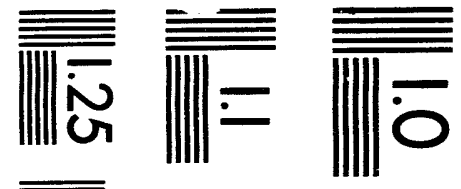

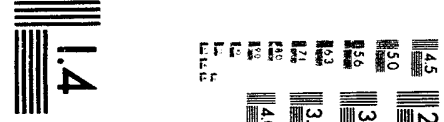

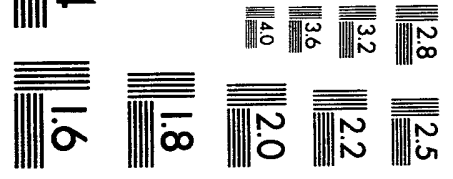



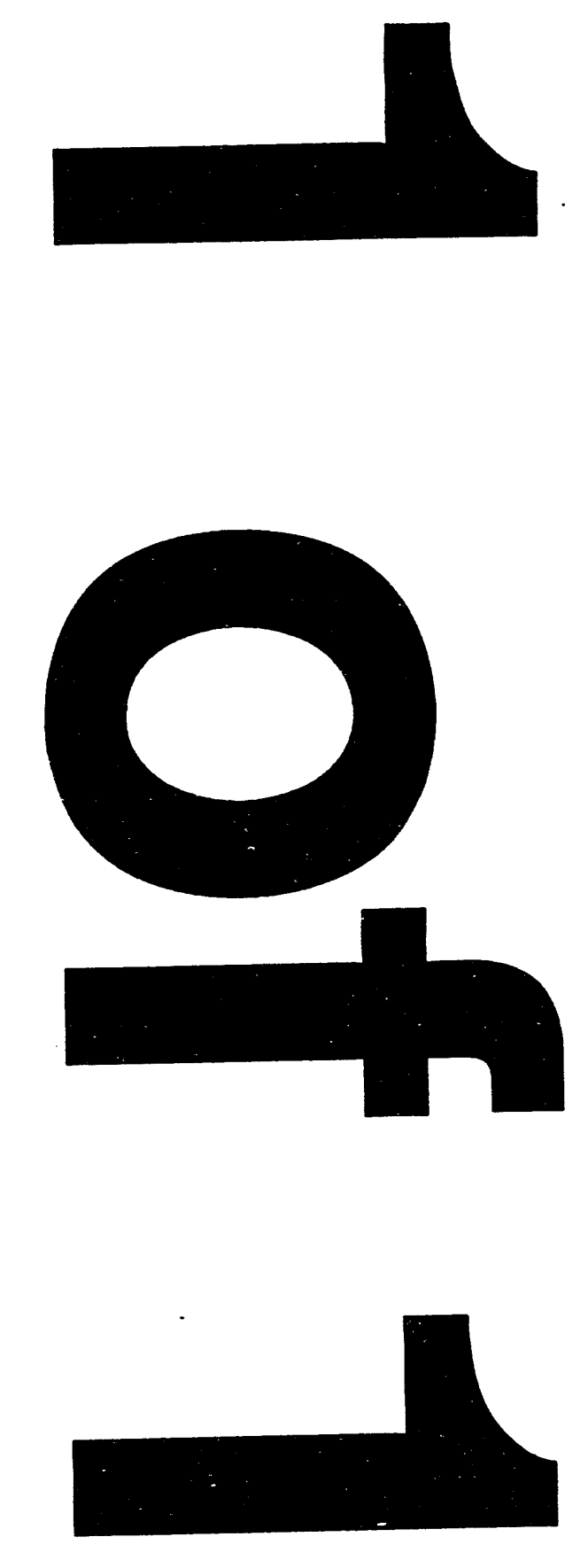


\title{
Inert Strength of Pristine Silica Glass Fibers
}

\author{
by
}

\author{
W. L. Smith and T. A. Michalske
}

Surface \& Interface Sciences

Sandia National Laboratories

Albuquerque, New Mexico 87185-0344

\begin{abstract}
Silica glass fibers have been produced and tested under ultra high vacuum (UHV) conditions to investigate the inert strength of pristine fibers in the absence of reactive agents. An analysis of the coefficient of variation in diameter $\left(\mathrm{U}_{\mathrm{d}}\right)$ vs the coefficient of variation of breaking strength $\left(v_{\sigma}\right)$ does not adequately explain the variation of breaking stress. The distribution of fiber tensile strength data suggests that the inert strength of such fibers is not single valued and that the intrinsic strength is controlled by defects in the glass. Furthermore, the comparison of room temperature UHV data with $\mathrm{LN}_{2}$ data indicates that these intrinsic strengths are not temperature dependent.
\end{abstract}

\section{MASTER}




\section{Introduction}

Silica based optical waveguides are rapidly assuming a pivotal role in advanced energy and defense related technologies. In many cases, the reliability of the entire fiber optic system is controlled by the mechanical properties of the silica glass fiber. As part of an effort to understand the mechanical properties of high purity glass fibers, it is necessary to examine the strength of pristine fibers that have not been exposed to abrasive contact or chemically reactive agents. Studies of high strength silica glass fibers in the pristine condition allow us to assess the potential of optical fibers and provide a basis for the examination, in a controlled fashion, of the role of chemical species in degrading fiber strength.

\section{Intrinsic \& Extrinsic Strength}

The intrinsic strength of a silica glass fiber is a measure of the perfection of the glass surface and of the glass structure. Since the flaws which control this strength are too small for observation, the intrinsic fracture strength is also used as an estimate of the distribution of these flaws. The extrinsic strength of silica fibers is controlled by macroscopic flaws such as abrasions and oxide contaminants which influence strength behavior. This reduction in strength is observed as a slope transition from intrinsic strength values on a Weibull distribution plot.

An additional factor which comes to play in evaluating strength behavior is the environment in which the glass fiber is fractured. Fibers are commonly tested in room temperature laboratory air which contains a substantial percentage of water vapor. Under these conditions the stress corrosion effects of adsorbed surface water predicate a distribution of strengths in the range of $3 G P a$ to $6 G P a^{(1,2)}$ which is well below the average strengths for pristine fibers which were observed in this study.

While it is clear that the fatigue behavior of low strength fibers is controlled by extrinsic flaws and reactive chemical species, little information is available which relates to the behavior of much higher strength pristine fibers. By producing and testing fibers relatively free from the effects of external strength degradation, we are able to investigate a higher range of strengths.

\section{Experimental}

In order to form contamination-free surfaces, we use an ultra high vacuum (UHV) chamber as a means of excluding particulate and chemical species during the fabrication procedure ${ }^{(3)}$. Short $(<3.0 \mathrm{~mm})$ sections of silica fiber are drawn within the vacuum chamber by means of a $\mathrm{CO}_{2}$ laser operating at a wavelength of $10.2 \mu \mathrm{m}$. After formation, fibers were tested to failure in a vacuum of $<5 \times 10^{-8}$ torr at a rate of $\approx 1.0 \mathrm{~g} / \mathrm{s}$. The strength data was fit to a two parameter Weibull distribution. Since fiber drawing and testing are conducted in situ, the handling and fixturing problems associated with delicate fiber sections are eliminated.

Strength data was accumulated for two grades of Amersil(4) fused silica - fused quartz materials. T-22 Suprasil-W1 is an extremely high purity fused silica which is characterized by its low bubble content, low inclusion content, and low $\mathrm{OH}$ content $(<5 \mathrm{ppm})$. T-08 Commercial is a lower grade fused quartz material with higher levels of bubbles, inclusions, and $\mathrm{OH}(\approx 180 \mathrm{ppm})$. T-22 is plasma fused from high purity synthetic materials $\left(\mathrm{SiCl}_{4}\right)$ and $\mathrm{T}-08$ is flame fused from natural crystalline quartz.

\section{Fiber Diameter Variation}

It has been previously shown(5) that the apparent variability in strength for fibers of a given uniformity

$\begin{array}{cc}\text { Fiber Diameter }(\mu m) & \text { Strength }(M P a) \\ 34.7 & 6609 \\ 34.5 & 6889 \\ 34.2 & 9736 \\ 34.6 & 10085 \\ 35.1 & 10098 \\ 33.9 & 10258 \\ 34.3 & 10638 \\ 34.7 & 10998 \\ 34.8 & 11302 \\ 34.5 & 11450 \\ 34.1 & 11703 \\ 34.1 & 12122 \\ 34.6 & 14103 \\ 34.5 & 14121 \\ 34.1 & 15127 \\ & \\ \text { Mean: } 34.58 \mu m & \text { Mean: } 11015 M P a \\ \sigma: 0.47988 \mu m & \sigma: 2360 M P a \\ v_{\mathrm{d}}: 0.0139 & v_{\sigma}: 0.215\end{array}$

Table I Fiber diameters and fiber strengths

of diameter is explained by a relationship between the measured coefficient of variation in fiber breaking 
strength $\left(v_{\sigma}\right)$ and the coefficient of variation in measured fiber diameter $\left(v_{d}\right)$.

$$
v_{\sigma}=2 v_{d}
$$

This analysis indicates that the observed variation of strength is due to variations in diameter measurement which relate directly to the cross section (area) of the fiber and that the true strength of the fibers are single valued. An analysis of the coefficient of variation of diameter for a representative fiber vs observed breaking strength variations for a pristine fiber of Amersil T-22 Suprasil-W is shown in Table I. While the coefficient of variation of fiber diameter is $1.4 \%$, the coefficient of variation of fiber strength is $21.5 \%$.

$$
v_{\sigma}=15 v_{d}
$$

The magnitude of this relationship suggests that the strength values are not single valued and that a more detailed analysis is required to explain the behavior of high strength pristine silica glass fibers.

\section{Inert Strength Distributions}

Figure 1 shows strength distribution for T-22 glass in ultra high vacuum (UHV) conditions at room temperature. The lower sample of data show relatively few points associated with extrinsic effects and a tight distribution of intrinsic values with a strength of $10 \mathrm{GPa}$ and a slope of 8 . In addition to this, there is another distribution of data with a nominal strength of $14 G P a$. This upper data sample clearly departs from the center sample of data and exhibits a slope of over 60 .

Figure 2 demonstrates a similar behavior for $\mathrm{T}$ 08 glass. In this case the slope values are somewhat

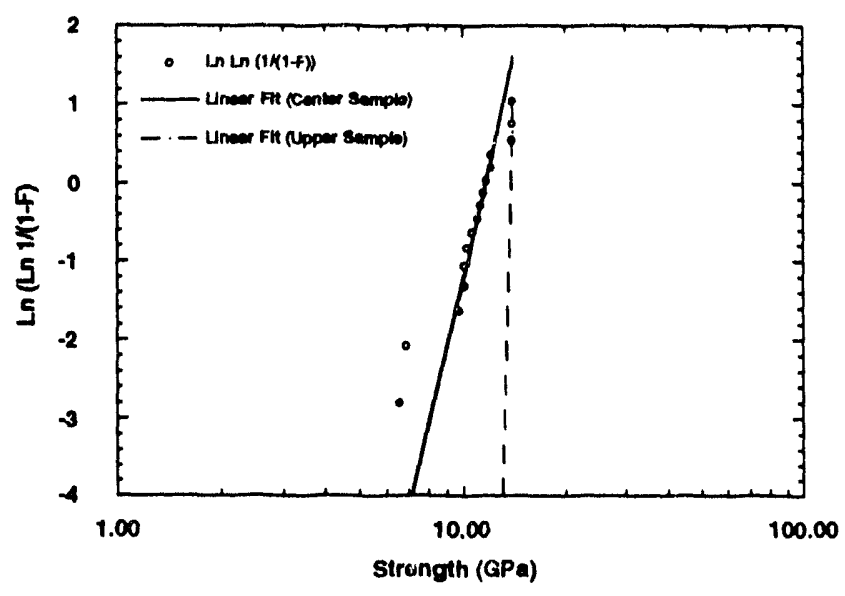

Figure 1 Strength distributions for T-22 different and a transition zone is apparent between the two strength distributions. Again, we see a distribution of strengths, in this case at $9 G P a$ and $14 G P a$. This type

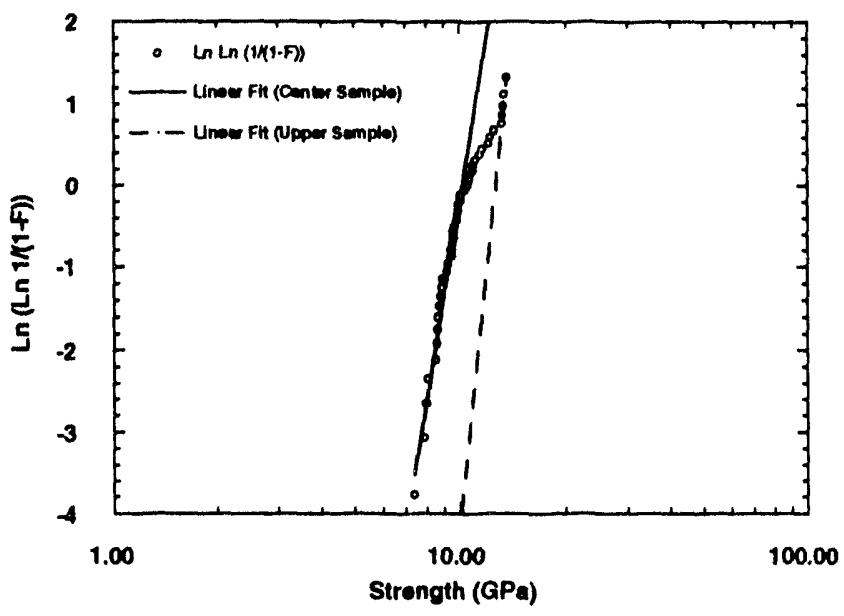

Figure 2 Strength distributions for T-08

of bimodal strength behavior has been shown previously as is seen in Hillig's( ${ }^{(6)}$ data in Figure 3. In this data, with silica fibers tested at $78^{\circ} \mathrm{K}$, there are distributions at 5 $G P a$ and again at $8 \mathrm{GPa}$. While none of our strength

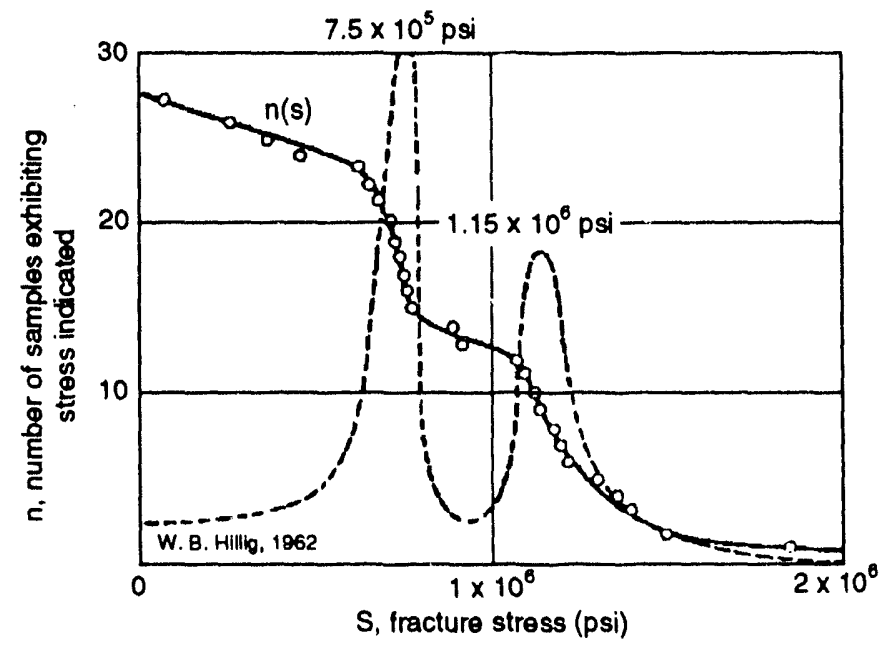

Figure 3 Strength distributions at $\mathrm{LN}_{2}$ temperature

values are as low as the $5 G P a$ distribution in Figure 3, our distribution at $9 \mathrm{GPa}$ agrees favorably with Hillig's findings and our value of $14 G P a$ for room temperature UHV strength is similar to his $\mathrm{LN}_{2}$ value of $13.6 \mathrm{GPa}$.

\section{Discussion}

Initial fiber tensile strength values show a disproportionate ratio between the coefficient of variation 
for fiber diameter $\left(v_{d}\right) v s$ coefficient of variation for fiber strength $\left(v_{\sigma}\right)$. While this analysis does not adequately explain the behavior of high strength pristine silica glass fibers, it is useful as a quality control tool in cases where environmental effects and surface condition, are the controlling mechanisms in fiber strength. This is partially due to the hydration of the extremely hydrophobic silica fiber surface as it cools from the drawing process in an uncontrolled atmosphere. When the fiber is eventually stressed in the presence of water the stress corrosion process $(7)$ progresses since any flaw can serve as an initiation site. These flaws may take the form of surface inclusions or contaminants, regions of constrained bonding in the random $\mathrm{SiO}_{2}$ structure, or that of surface vacancy concentrations produced during the rapid quenching of the fiber.

As we have seen in the comparison of T-22 and T08 fibers which were produced and tested under very well controlled conditions, there is an apparent compositional effect shown by the variation in strength distributions for the two materials. Given the wide variation of $\mathrm{SiO}_{2}$ materials and testing conditions, it is not unreasonable to see some variation in the absolute placement of these distributions when compared with other work. Of greater interest, is the appearance of the additional distribution at the high end of the strength data. This behavior does not indicate that the inert strength is single valued and further suggests that the strength behavior is due to some defect in the glass.

Intrinsic fiber strength data taken at room temperature in ultra high vacuum conditions compares favorably with fiber strength data taken at $78^{\circ} \mathrm{K}$. Inert strengths on the order of $14 \mathrm{GPa}$ have been measured with both techniques indicating that the behavior is not temperature dependent but is related to the absence of chemical reactants at the glass surface.

\section{References}

1. U. C. Paek, C. D. Spainhour, C. M. Schroeder, \& C. R. Kurkjian, "Tensile Strength of 50-m Long Silica Fibers Drawn with a Galvanometer Scanning System", J. Am. Ceram. Soc., Vol. 5\&, [6], 630-633, (1980).

2. W. J. Duncan, P. W. France, \& S. P. Craig, "The Effect of Environment on the Strength of Optical Fiber", in Strength of Inorganic Glass, Edited by C. R. Kurkjian, Plenum Press, NY, (1989).

3. W. L. Smith, "Automated Glass Fiber Drawing", Sandia National Laboratories, SAND89-1674, (1989).

4. From Amersil Inc, brochure EM-9227-2, (no date).

5. C. R. Kurkjian \& U. C. Paek, "Single-valued strength of "perfect" silica fibers", Appl. Phys. Lett., 42, [3], (1983).

6. W. B. Hillig, "The Factors Affecting the Ultimate Strength of Bulk Fused Silica", in Symposium sur la Resistance Mecanique du Verre et les Moyens de l'ameliorer, Union Scientific Contientale du Verre, 295325, (1962).

7. T. A. Michalske \& B. C. Bunker, "Slow fracture model based on strained silicate structıres", J. Appl. Phys., 56, [10], (1984). 
DISTRIBUTION:

$\begin{array}{llrl}\text { MS 0344 } & 1114 & 1 & \text { T. A. Michalske } \\ \text { MS 0344 } & 1114 & 20 & \text { W. L. Smith } \\ \text { MS 0899 } & 7141 & 5 & \text { Technical Library } \\ \text { MS 0619 } & 7151 & 1 & \text { Technical Publications } \\ \text { MS 1119 } & 7613-2 & 10 & \text { Document Processing } \\ \text { MS 9018 } & 8523-2 & 1 & \text { Central Technical Files }\end{array}$



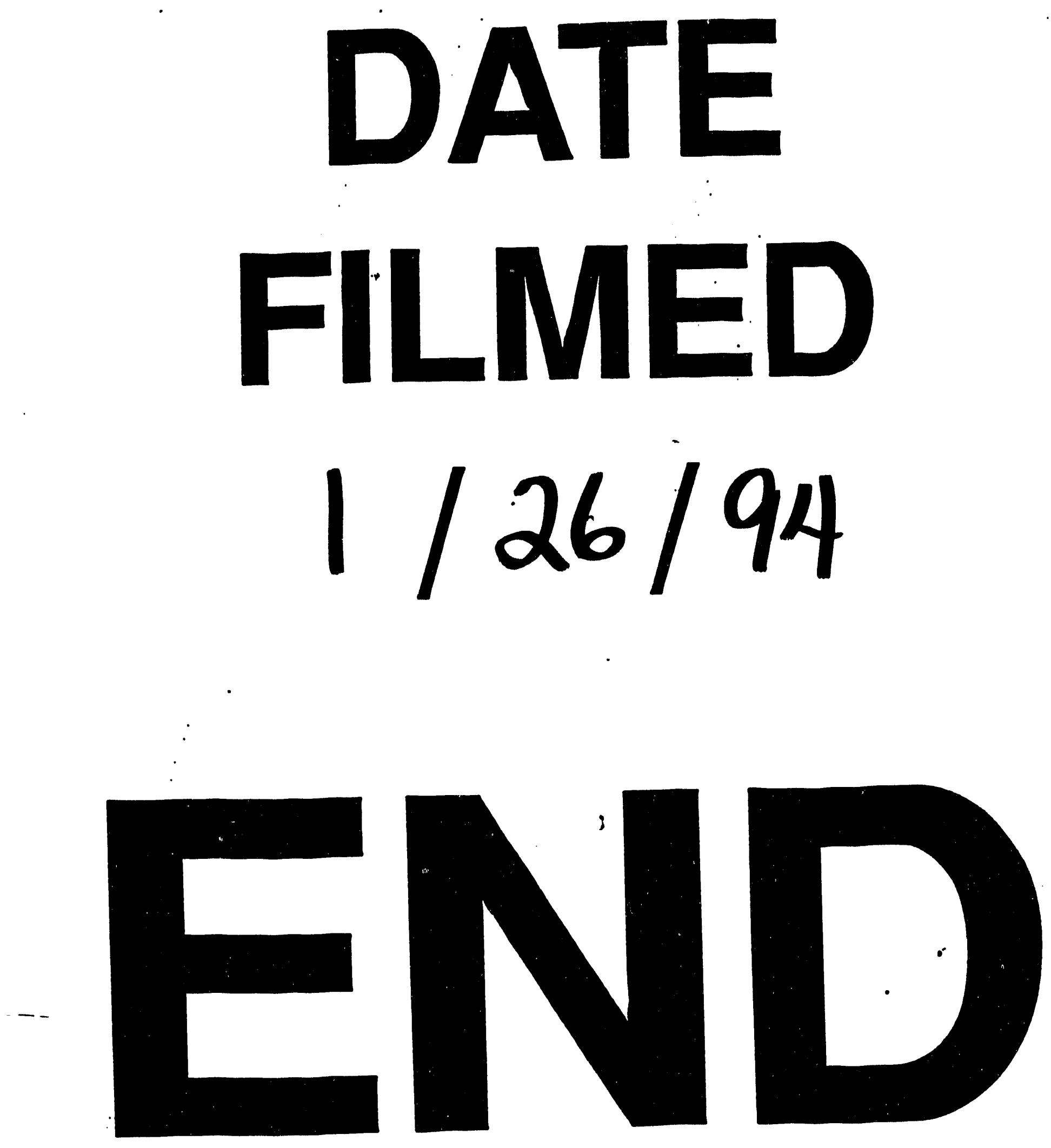


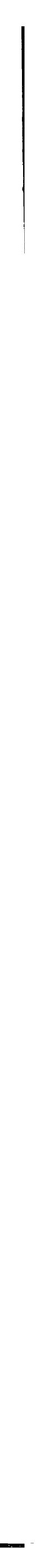

